Yukawa Institute Kyoto

YITP-03-06

hep-th/0303077

March 2003

\title{
Algebraic Bethe ansatz for the elliptic quantum group $E_{\tau, \eta}\left(s l_{n}\right)$ and its applications
}

\author{
B. Y. Hou ${ }^{a}$, R. Sasaki ${ }^{b}$ and W.-L. Yang ${ }^{a, b}$ \\ ${ }^{a}$ Institute of Modern Physics, Northwest University \\ Xian 710069, P.R. China \\ ${ }^{b}$ Yukawa Institute for Theoretical Physics, \\ Kyoto University, Kyoto 606-8502, Japan
}

\begin{abstract}
We study the tensor product of the higher spin representations (see the definition in Sect. 2.2) of the elliptic quantum group $E_{\tau, \eta}\left(s l_{n}\right)$. The transfer matrices associated with the $E_{\tau, \eta}\left(s l_{n}\right)$-module are exactly diagonalized by the nested Bethe ansatz method. Some special cases of the construction give the exact solution for the $\mathbb{Z}_{n}$ Belavin model and for the elliptic $A_{n-1}$ Ruijsenaars-Schneider model.
\end{abstract}

PACS: 03.65.Fd; 05.30.-d

Keywords: Integrable models; Elliptic quantum group; Bethe ansatz; $\mathbb{Z}_{n}$ Belavin model; Ruijsenaars-Schneider model. 


\section{Introduction}

Bethe ansatz method has proved to be the most powerful and (probably) unified method to construct the common eigenvectors of commuting families of operators (usually called transfer matrices) in two-dimensional integrable models [1, 2, 3]. Faddeev et al [1] reformulated the Bethe ansatz method in a representation theory form: transfer matrices are associated with representations of certain algebras with quadratic relations (now called quantum groups). The eigenvectors are constructed by acting certain algebra elements on the " highest weight vectors". This type of Bethe ansatz is known as the algebraic Bethe ansatz.

Whereas this construction has been very successful in rational and trigonometric integrable models [4, 5], its extension to elliptic models had been problematic due to the fact that for the underlying algebras $-\mathbb{Z}_{n}$ Sklyanin algebras [6, 7] the highest weight representations were not properly defined. Therefore, the algebraic Bethe ansatz method had not be applied to the elliptic integrable models directly.

Recently, a definition of elliptic quantum groups $E_{\tau, \eta}(\mathfrak{g})$ associated with any simple classical Lie algebra $\mathfrak{g}$ was given [8]. The highest weight representations of the elliptic quantum groups (cf. $\mathbb{Z}_{n}$ Sklyanin algebras) are now well-defined [8, 9]. This enabled Felder et al [10] to apply successfully the algebraic Bethe ansatz method for constructing the eigenvectors of the transfer matrices of the elliptic integrable models associated with modules over $E_{\tau, \eta}\left(s l_{2}\right)$, and Billey [11] to apply the algebraic nested Bethe ansatz method for constructing the eigenvalues of the transfer matrices associated with a special module over $E_{\tau, \eta}\left(s l_{n}\right)$ (see section $5)$.

In this paper, we will extend the above construction of Bethe ansatz method further to the elliptic quantum group $E_{\tau, \eta}\left(s l_{n}\right)$ on a generic higer spin module $W=V_{\Lambda^{\left(l_{1}\right)}}\left(z_{1}\right) \otimes$ $V_{\Lambda^{\left(l_{2}\right)}}\left(z_{2}\right) \otimes \cdots \otimes V_{\Lambda^{\left(l_{m}\right)}}\left(z_{m}\right)$. After briefly reviewing the definition of the elliptic quantum group $E_{\tau, \eta}\left(s l_{n}\right)$, we study one parameter series of highest weight representations (higher spin representations) of $E_{\tau, \eta}\left(s l_{n}\right)$, and introduce the associated operator algebra and the transfer matrices corresponding to the $E_{\tau, \eta}\left(s l_{n}\right)$-module in section 2 . In section 3 , we describe the algebraic Bethe ansatz for $E_{\tau, \eta}\left(s l_{n}\right)$. Finally, we give som e applications of our construction to the $\mathbb{Z}_{n}$ Belavin models in section 4, and the elliptic Ruijsenaars-Schneider model associated to $A_{n-1}$ root system in section 5 . 


\section{The elliptic quantum group and its modules associ- ated to $A_{n-1}$}

\subsection{The elliptic quantum group associated to $A_{n-1}$}

We first review the definition of the elliptic quantum group $E_{\tau, \eta}\left(s l_{n}\right)$ associated to $A_{n-1}$ 8. Let $\left\{\epsilon_{i} \mid i=1,2, \cdots, n\right\}$ be the orthonormal basis of the vector space $\mathbb{C}^{n}$ such that $\left\langle\epsilon_{i}, \epsilon_{j}\right\rangle=\delta_{i j}$. The $A_{n-1}$ simple roots are $\left\{\alpha_{i}=\epsilon_{i}-\epsilon_{i+1} \mid i=1, \cdots, n-1\right\}$ and the fundamental weights $\left\{\Lambda_{i} \mid i=1, \cdots, n-1\right\}$ satisfying $\left\langle\Lambda_{i}, \alpha_{j}\right\rangle=\delta_{i j}$ are given by

$$
\Lambda_{i}=\sum_{k=1}^{i} \epsilon_{k}-\frac{i}{n} \sum_{k=1}^{n} \epsilon_{k} .
$$

Set

$$
\hat{i}=\epsilon_{i}-\bar{\epsilon}, \quad \bar{\epsilon}=\frac{1}{n} \sum_{k=1}^{n} \epsilon_{k}, \quad i=1, \cdots, n, \quad \text { then } \sum_{i=1}^{n} \hat{i}=0 .
$$

For each dominant weight $\Lambda=\sum_{i=1}^{n-1} a_{i} \Lambda_{i}, \quad a_{i} \in \mathbb{Z}^{+}$, there exists an irreducible highest weight finite-dimensional representation $V_{\Lambda}$ of $A_{n-1}$ with the highest vector $|\Lambda\rangle$. For example the fundamental vector representation is $V_{\Lambda_{1}}$. In this paper, we only consider the symmetric tensor-product representation of $\overbrace{V_{\Lambda_{1}} \otimes V_{\Lambda_{1}} \cdots \otimes V_{\Lambda_{1}}}^{l}$ (or, the higher spin-l representation of $\left.A_{n-1}\right)$, namely, the one parameter series of highest weight representations $V_{\Lambda^{(l)}}$, with

$$
\Lambda^{(l)}=l \Lambda_{1}, \quad l \in \mathbb{Z} \text { and } l>0 .
$$

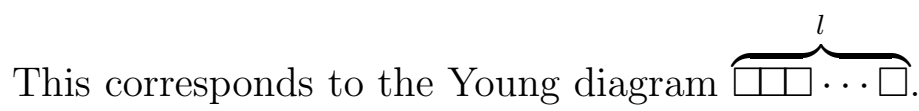

Let $\mathfrak{h}$ be the Cartan subalgebra of $A_{n-1}$ and $\mathfrak{h}^{*}$ be its dual. A finite dimensional diagonalizable $\mathfrak{h}$-module is a complex finite dimensional vector space $W$ with a weight decomposition $W=\oplus_{\mu \in \mathfrak{h}^{*}} W[\mu]$, so that $\mathfrak{h}$ acts on $W[\mu]$ by $x v=\mu(x) v,(x \in \mathfrak{h}, v \in W[\mu])$. For example, the fundamental vector representation $V_{\Lambda_{1}}=\mathbb{C}^{n}$, the non-zero weight spaces are $W[\hat{i}]=\mathbb{C} \epsilon_{i}, i=1, \cdots, n$.

Let us fix $\tau$ such that $\operatorname{Im}(\tau)>0$ and a generic complex number $\eta$. For convenience, we introduce another parameter $w=n \eta$ related to $\eta$. Let us introduce the following elliptic functions

$$
\sigma(u)=\theta\left[\begin{array}{c}
\frac{1}{2} \\
\frac{1}{2}
\end{array}\right](u, \tau), \quad \theta^{(j)}(u)=\theta\left[\begin{array}{c}
\frac{1}{2}-\frac{j}{n} \\
\frac{1}{2}
\end{array}\right](u, n \tau),
$$




$$
\theta\left[\begin{array}{l}
a \\
b
\end{array}\right](u, \tau)=\sum_{m=-\infty}^{\infty} \exp \left\{\sqrt{-1} \pi\left[(m+a)^{2} \tau+2(m+a)(u+b)\right]\right\}
$$

For a generic complex weight $\xi \in \sum_{i=1}^{n-1} \mathbb{C} \Lambda_{i}$, we introduce a weight lattice with a shift by $\xi, \mathbb{P}_{\xi}$ as follows

$$
\mathbb{P}_{\xi}=\xi+\sum_{i=1}^{n} m_{i} \hat{i}, \quad m_{i} \in \mathbb{Z}
$$

For $\lambda \in \mathbb{P}_{\xi}$, define

$$
\lambda_{i}=\left\langle\lambda, \epsilon_{i}\right\rangle, \quad \lambda_{i j}=\lambda_{i}-\lambda_{j}=\left\langle\lambda, \epsilon_{i}-\epsilon_{j}\right\rangle, \quad i, j=1, \cdots, n
$$

In this paper, we restrict $\lambda \in \mathbb{P}_{\xi}$ so that the inverse of the matrix $S(z, \lambda)$ in (4.7) does exist. Let $R(z, \lambda) \in \operatorname{End}\left(\mathbb{C}^{n} \otimes \mathbb{C}^{n}\right)$ be the R-matrix given by

$$
R(z, \lambda)=\sum_{i=1}^{n} R_{i i}^{i i}(z, \lambda) E_{i i} \otimes E_{i i}+\sum_{i \neq j}\left\{R_{i j}^{i j}(z, \lambda) E_{i i} \otimes E_{j j}+R_{i j}^{j i}(z, \lambda) E_{j i} \otimes E_{i j}\right\},
$$

in which $E_{i j}$ is the matrix with elements $\left(E_{i j}\right)_{k}^{l}=\delta_{j k} \delta_{i l}$. The coefficient functions are

$$
\begin{aligned}
& R_{i i}^{i i}(z, \lambda)=1, \quad R_{i j}^{i j}(z, \lambda)=\frac{\sigma(z) \sigma\left(\lambda_{i j} w+w\right)}{\sigma(z+w) \sigma\left(\lambda_{i j} w\right)}, \\
& R_{i j}^{j i}(z, \lambda)=\frac{\sigma(w) \sigma\left(z+\lambda_{i j} w\right)}{\sigma(z+w) \sigma\left(\lambda_{i j} w\right)}
\end{aligned}
$$

and $\lambda_{i j}$ is defined in (2.6). The R-matrix satisfies the dynamical (modified) quantum YangBaxter equation

$$
\begin{aligned}
& R_{12}\left(z_{1}-z_{2}, \lambda-h^{(3)}\right) R_{13}\left(z_{1}-z_{3}, \lambda\right) R_{23}\left(z_{2}-z_{3}, \lambda-h^{(1)}\right) \\
& \quad=R_{23}\left(z_{2}-z_{3}, \lambda\right) R_{13}\left(z_{1}-z_{3}, \lambda-h^{(2)}\right) R_{12}\left(z_{1}-z_{2}, \lambda\right),
\end{aligned}
$$

with the initial condition

$$
R_{i j}^{k l}(0, \lambda)=\delta_{i}^{l} \delta_{j}^{k}
$$

We adopt the notation: $R_{12}\left(z, \lambda-h^{(3)}\right)$ acts on a tensor $v_{1} \otimes v_{2} \otimes v_{3}$ as $R(z ; \lambda-\mu) \otimes I d$ if $v_{3} \in W[\mu]$.

A representation of the elliptic quantum group $E_{\tau, \eta}\left(s l_{n}\right)$ (an $E_{\tau, \eta}\left(s l_{n}\right)$-module) is by definition a pair $(W, L)$ where $W$ is a diagonalizable $\mathfrak{h}$-module and $L(z, \lambda)$ is a meromorphic 
function of $\lambda$ and the spectral parameter $z \in \mathbb{C}$, with values in $\operatorname{End}_{\mathfrak{h}}\left(\mathbb{C}^{n} \otimes W\right.$ ) (the endomorphism commuting with the action of $\mathfrak{h}$ ). It obeys the so-called " $R L L$ " relation

$$
\begin{aligned}
& R_{12}\left(z_{1}-z_{2}, \lambda-h^{(3)}\right) L_{13}\left(z_{1}, \lambda\right) L_{23}\left(z_{2}, \lambda-h^{(1)}\right) \\
& \quad=L_{23}\left(z_{2}, \lambda\right) L_{13}\left(z_{1}, \lambda-h^{(2)}\right) R_{12}\left(z_{1}-z_{2}, \lambda\right)
\end{aligned}
$$

where the first and second space are auxiliary spaces $\left(\mathbb{C}^{n}\right)$ and the third space plays the role of quantum space $(W)$. The total weight conservation condition for the $L$-operator reads

$$
\left[h^{(1)}+h^{(3)}, L_{13}(z, \lambda)\right]=0 .
$$

In terms of the elements of the $L$-operator defined by

$$
L(z, \lambda)\left(e_{i} \otimes v\right)=\sum_{j=1}^{n} e_{j} \otimes L_{i}^{j}(z, \lambda) v, \quad v \in W
$$

the above condition can be expressed equivalently as

$$
f(h) L_{i}^{j}(z, \lambda)=L_{i}^{j}(z, \lambda) f(h+\hat{i}-\hat{j})
$$

in which $f(h)$ is any meromorphic function of $h$ and $h$ measures the weight of the quantum space $(W)$.

\subsection{Modules over $E_{\tau, \eta}\left(s l_{n}\right)$ and the associated operator algebra}

The basic example of an $E_{\tau, \eta}\left(s l_{n}\right)$-module is $\left(\mathbb{C}^{n}, L\right)$ with $L(z, \lambda)=R\left(z-z_{1}, \lambda\right)$, which is called the fundamental vector representation $V_{\Lambda_{1}}\left(z_{1}\right)$ with the evaluation point $z_{1}$. It is obvious that " $R L L$ " relation is satisfied as a consequence of the dynamical Yang-Baxter equation (2.10). Other modules can be obtained by taking tensor products: if $\left(W_{1}, L^{(1)}\right)$ and $\left(W_{2}, L^{(2)}\right)$ are $E_{\tau, \eta}\left(s l_{n}\right)$-modules, where $L^{(j)}$ acts on $\left(\mathbb{C}^{n} \otimes W_{j}\right)$, then also $\left(W_{1} \otimes W_{2}, L\right)$ with

$$
L(z, \lambda)=L^{(1)}\left(z, \lambda-h^{(2)}\right) L^{(2)}(z, \lambda) \text { acting on } \mathbb{C}^{n} \otimes W_{1} \otimes W_{2} .
$$

An $E_{\tau, \eta}\left(s l_{n}\right)$-submodule of an $E_{\tau, \eta}\left(s l_{n}\right)$-module $(W, L)$ is a pair $\left(W_{1}, L_{1}\right)$ where $W_{1}$ is an $\mathfrak{h}$-submodule of $W$ such that $\mathbb{C}^{n} \otimes W_{1}$ is invariant under the action of all the $L(z, \lambda)$, and $L_{1}(z, \lambda)$ is the restriction to this invariant subspace. Namely, the $E_{\tau, \eta}\left(s l_{n}\right)$-submodules are $E_{\tau, \eta}\left(s l_{n}\right)$-modules. 
Using the fusion rule of $E_{\tau, \eta}\left(s l_{n}\right)$ (2.15) one can construct the symmetric $E_{\tau, \eta}\left(s l_{n}\right)$ submodule of $l$-tensors of fundamental vector representations:

$$
V_{\Lambda^{(l)}}\left(z_{1}\right)=\text { symmetric subspace of } V_{\Lambda_{1}}\left(z_{1}\right) \otimes V_{\Lambda_{1}}\left(z_{1}-w\right) \otimes \cdots \otimes V_{\Lambda_{1}}\left(z_{1}-(l-1) w\right) \text {, }
$$

where $\Lambda^{(l)}$ is defined by (2.2). We call such an $E_{\tau, \eta}\left(s l_{n}\right)$-module the higher spin-l representation with evaluation point $z_{1}$. These series of representations in the case of $\mathbb{Z}_{n}$ Sklyanin algebra have been studied in [6] for $n=2$ case and in [12, 13] for generic $n$ case. From direct calculation, we find that the $E_{\tau, \eta}\left(s l_{n}\right)$-module $V_{\Lambda^{(l)}}(z)$ is an irreducible highest weight module of $E_{\tau, \eta}\left(s l_{n}\right)$ with the highest vector $\left|\Lambda^{(l)}\right\rangle \in W\left[l \Lambda_{1}\right]=\mathbb{C}\left|\Lambda^{(l)}\right\rangle$. It satisfies the following highest weight conditions

$$
\begin{aligned}
& L_{1}^{1}(u, \lambda)\left|\Lambda^{(l)}\right\rangle=\left|\Lambda^{(l)}\right\rangle, \quad L_{1}^{i}(u, \lambda)\left|\Lambda^{(l)}\right\rangle=0, \quad i=2, \cdots, n, \\
& L_{j}^{i}(u, \lambda)\left|\Lambda^{(l)}\right\rangle=\delta_{j}^{i} \frac{\sigma(u-z) \sigma\left(\lambda_{i 1} w+l w\right)}{\sigma(u-z+l w) \sigma\left(\lambda_{i 1} w\right)}\left|\Lambda^{(l)}\right\rangle, \quad i, j=2, \cdots, n, \\
& f(h)\left|\Lambda^{(l)}\right\rangle=f(l \hat{1})\left|\Lambda^{(l)}\right\rangle,
\end{aligned}
$$

where $f(h)$ is any meromorphic function of $h$, which measures the weight of the quantum space $W$.

For any $E_{\tau, \eta}\left(s l_{n}\right)$-module, as in [10] one can define an associated operator algebra of difference operators on the space $\operatorname{Fun}(W)$ of meromorphic functions of $\lambda \in \mathbb{P}_{\xi}$ with values in $W$. The algebra is generated by $h$ and the operators $\tilde{L}(z) \in \operatorname{End}\left(\mathbb{C}^{n} \otimes F u n(W)\right)$ acting as

$$
\tilde{L}(z)\left(e_{i} \otimes f\right)(\lambda)=\sum_{j=1}^{n} e_{j} \otimes L_{i}^{j}(z, \lambda) f(\lambda-\hat{i})
$$

One can derive the following exchange relation of the difference operator $\tilde{L}(z)$ from the "RLL" relation (2.12), the weight conservation condition of $L_{i}^{j}(z, \lambda)$ (2.14) and the fact $\left[h^{(1)}+h^{(2)}, R_{12}(z, \lambda)\right]=0$,

$$
\begin{aligned}
& R_{12}\left(z_{1}-z_{2}, \lambda-h\right) \tilde{L}_{13}\left(z_{1}\right) \tilde{L}_{23}\left(z_{2}\right)=\tilde{L}_{23}\left(z_{2}\right) \tilde{L}_{13}\left(z_{1}\right) R_{12}\left(z_{1}-z_{2}, \lambda\right), \\
& f(h) \tilde{L}_{i}^{j}(z)=\tilde{L}_{i}^{j}(z) f(h+\hat{i}-\hat{j})
\end{aligned}
$$

where $f(h)$ is any meromorphic function of $h$ and $h$ measures the weight of the quantum space $W$. 
The transfer matrices associated to an $E_{\tau, \eta}\left(s l_{n}\right)$-module $(W, L)$ [10] is a difference operator acting on the space $\operatorname{Fun}(W)[0]$ of meromorphic functions of $\lambda$ with values in the zero-weight space of $W$. It is defined by

$$
T(u) f(\lambda)=\sum_{i=1}^{n} \tilde{L}_{i}^{i}(u) f(\lambda)=\sum_{i=1}^{n} L_{i}^{i}(u, \lambda) f(\lambda-\hat{i}) .
$$

The exchange relation of $\tilde{L}$-operators (2.20) and (2.21) imply that, for any $E_{\tau, \eta}\left(s l_{n}\right)$-module, the above transfer matrices preserve the space $H=F u n(W)[0]$ of functions with values in the zero weight space $W[0]$. Moreover, they commute pairwise on $H$ :

$$
\left.[T(u), T(v)]\right|_{H}=0 .
$$

In this paper we will study the tensor product $E_{\tau, \eta}\left(s l_{n}\right)$-module $W=V_{\Lambda^{\left(l_{1}\right)}}\left(z_{1}\right) \otimes$ $V_{\Lambda^{\left(l_{2}\right)}}\left(z_{2}\right) \otimes \cdots \otimes V_{\left.\Lambda^{(l m}\right)}\left(z_{m}\right)$. With the generic evaluation points $\left\{z_{i}\right\}$, the module is an irreducible highest weight $E_{\tau, \eta}\left(s l_{n}\right)$-module $[8,9]$. Let $\Lambda=\Lambda^{\left(l_{1}\right)}+\cdots+\Lambda^{\left(l_{m}\right)}$, then $W[\Lambda]=\mathbb{C}|\Lambda\rangle$ with

$$
|\Lambda\rangle \equiv\left|\Lambda^{\left(l_{1}\right)}, \Lambda^{\left(l_{2}\right)}, \cdots, \Lambda^{\left(l_{m}\right)}\right\rangle \equiv\left|\Lambda^{\left(l_{1}\right)}\right\rangle \otimes\left|\Lambda^{\left(l_{2}\right)}\right\rangle \otimes \cdots \otimes\left|\Lambda^{\left(l_{m}\right)}\right\rangle .
$$

The vector $|\Lambda\rangle$, viewed as a constant function in $F u n(W)$, obeys the following highest weight conditions:

$$
\begin{aligned}
& \tilde{L}_{1}^{1}(z)|\Lambda\rangle=A(z, \lambda)|\Lambda\rangle, \quad \tilde{L}_{1}^{i}(z)|\Lambda\rangle=0, \quad i=2, \cdots, n \\
& \tilde{L}_{j}^{i}(z)|\Lambda\rangle=\delta_{j}^{i} D_{i}(z, \lambda)|\Lambda\rangle, \quad i, j=2, \cdots, n, \quad f(h)|\Lambda\rangle=f(N \hat{1})|\Lambda\rangle .
\end{aligned}
$$

The highest weight functions read

$$
A(z, \lambda)=1, \quad D_{i}(z, \lambda)=\left\{\prod_{k=1}^{m} \frac{\sigma\left(z-p_{k}\right)}{\sigma\left(z-q_{k}\right)}\right\} \frac{\sigma\left(\lambda_{i 1} w+N w\right)}{\sigma\left(\lambda_{i 1} w\right)}, \quad i=2, \cdots, n,
$$

where

$$
p_{k}=z_{k}, \quad q_{k}=z_{k}-l_{k} w, \quad N=\sum_{k=1}^{m} l_{k}, \quad k=1, \cdots, m
$$

\section{Algebraic Bethe ansatz for $E_{\tau, \eta}\left(s l_{n}\right)$}

In this section we fix a highest weight $E_{\tau, \eta}\left(s l_{n}\right)$-module $W$ of weight $\Lambda$, the functions $A(z, \lambda), D_{i}(z, \lambda)$ (2.24), with the highest vector $|\Lambda\rangle$. We assume that $N=\sum_{k=1}^{m} l_{k}=n \times l$ 
with $l$ being an integer, so that the zero-weight space $W[0]$ can be non-trivial and so that the algebraic Bethe ansatz method can be applied as in [14, 15, 16, 10].

Let us adopt the standard notation for convenience:

$$
\begin{aligned}
& \mathcal{A}(u)=\tilde{L}_{1}^{1}(u), \quad \mathcal{B}_{i}(u)=\tilde{L}_{i}^{1}(u), \quad i=2, \cdots, n, \\
& \mathcal{C}_{i}(u)=\tilde{L}_{1}^{i}(u), \quad \mathcal{D}_{i}^{j}(u)=\tilde{L}_{i}^{j}(u), \quad i, j=2, \cdots, n .
\end{aligned}
$$

The transfer matrices $T(u)$ become

$$
T(u)=\mathcal{A}(u)+\sum_{i=2}^{n} \mathcal{D}_{i}^{i}(u) .
$$

Any non-zero vector $|\Omega\rangle \in F u n(W)[\Lambda]$ is of form $|\Omega\rangle=g(\lambda)|\Lambda\rangle$, for some meromorphic function $g \neq 0$. When $N=n \times l$, the weight $\Lambda$ can be written in the form

$$
\Lambda=n l \Lambda_{1}=l \sum_{k=1}^{n-1}\left(\epsilon_{1}-\epsilon_{k+1}\right) .
$$

Noting (2.21), the zero-weight vector space is spanned by the vectors of the following form

$$
\mathcal{B}_{i_{N_{1}}}\left(v_{N_{1}}\right) \mathcal{B}_{i_{N_{1}-1}}\left(v_{N_{1}-1}\right) \cdots \mathcal{B}_{i_{1}}\left(v_{1}\right)|\Omega\rangle
$$

where $N_{1}=(n-1) \times l$ and among the indices $\left\{i_{k} \mid k=1, \cdots, N_{1}\right\}$, the number of $i_{k}=j$, denoted by $\#(j)$, should be

$$
\#(j)=l, \quad j=2, \cdots, n .
$$

The above states (3.5) actually belong to the zero-weight space $W[0]$, because

$$
\begin{aligned}
f & (h) \mathcal{B}_{i_{N_{1}}}\left(v_{N_{1}}\right) \mathcal{B}_{i_{N_{1}-1}}\left(v_{N_{1}-1}\right) \cdots \mathcal{B}_{i_{1}}\left(v_{1}\right)|\Omega\rangle \\
& =\mathcal{B}_{i_{N_{1}}}\left(v_{N_{1}}\right) \mathcal{B}_{i_{N_{1}-1}}\left(v_{N_{1}-1}\right) \cdots \mathcal{B}_{i_{1}}\left(v_{1}\right) f\left(h+\sum_{k=1}^{N_{1}} \hat{i}_{k}-N_{1} \hat{1}\right)|\Omega\rangle \\
& =f\left(l \sum_{i=2}^{n} \hat{i}-(n-1) l \hat{1}+n l \hat{1}\right) \mathcal{B}_{i_{N_{1}}}\left(v_{N_{1}}\right) \mathcal{B}_{i_{N_{1}-1}}\left(v_{N_{1}-1}\right) \cdots \mathcal{B}_{i_{1}}\left(v_{1}\right)|\Omega\rangle \\
& =f(0) \mathcal{B}_{i_{N_{1}}}\left(v_{N_{1}}\right) \mathcal{B}_{i_{N_{1}-1}}\left(v_{N_{1}-1}\right) \cdots \mathcal{B}_{i_{1}}\left(v_{1}\right)|\Omega\rangle
\end{aligned}
$$

for any meromorphic function $f$. We will seek the common eigenvectors of the transfer matrices $T(u)$ in the form

$$
\left|\lambda ;\left\{v_{k}\right\}\right\rangle=F^{i_{1}, i_{2}, \cdots, i_{N_{1}}}\left(\lambda ;\left\{v_{k}\right\}\right) \mathcal{B}_{i_{N_{1}}}\left(v_{N_{1}}\right) \mathcal{B}_{i_{N_{1}-1}}\left(v_{N_{1}-1}\right) \cdots \mathcal{B}_{i_{1}}\left(v_{1}\right)|\Omega\rangle,
$$


with the restriction condition (3.6) and the parameters $\left\{v_{k}\right\}$ will be specified later by the Bethe ansatz equations (3.46). We adopt here the convention that the repeated indices imply summation over $2,3, \cdots, n$, and the notation that

$$
v_{k}=v_{k}^{(0)}, k=1,2, \cdots, N_{1} .
$$

For convenience, let us introduce the following set of integers

$$
N_{i}=(n-i) \times l, \quad i=1,2, \cdots, n-1,
$$

and $(n-1)$ complex parameters $\left\{\alpha^{(i)} \mid i=1, \cdots, n-1\right\}$ and set $\alpha^{(n)}=-\sum_{k=1}^{n-1} \alpha^{(k)}$ to specify quasi-vacuums of each step of the nested Bethe ansatz (see below). Associated with $\left\{\alpha^{(i)}\right\}$, set

$$
\bar{\alpha}^{(i)}=\frac{1}{(n-i-1)}\left\{\alpha^{(i+1)}-\frac{\sum_{k=i+1}^{n} \alpha^{(k)}}{n-i}\right\}, \quad i=0, \cdots, n-2 .
$$

From the exchange relations (2.20) and (2.21), one can derive the commutation relations among $\mathcal{A}(u), \mathcal{D}_{i}^{j}(u)$ and $\mathcal{B}_{i}(u)(i, j=2, \cdots, n)$ (for details, see Appendix A). The relevant commutation relations are

$$
\begin{aligned}
\mathcal{A}(u) \mathcal{B}_{i}(v)= & r\left(v-u, \lambda_{i 1}\right) \mathcal{B}_{i}(v) \mathcal{A}(u)+s\left(u-v, \lambda_{1 i}\right) \mathcal{B}_{i}(u) A(v), \\
\mathcal{D}_{i}^{j}(u) \mathcal{B}_{l}(v)= & r\left(u-v, \lambda_{j 1}-h_{j 1}\right) \sum_{\alpha, \beta=2}^{n} R_{i l}^{\alpha \beta}(u-v, \lambda) \mathcal{B}_{\beta}(v) \mathcal{D}_{\alpha}^{j}(u) \\
& -s\left(u-v, \lambda_{1 j}-h_{1 j}\right) \mathcal{B}_{i}(u) \mathcal{D}_{l}^{j}(v), \\
\mathcal{B}_{i}(u) \mathcal{B}_{j}(v)= & \sum_{\alpha, \beta=2}^{n} R_{i}^{\alpha \beta}(u-v, \lambda) \mathcal{B}_{\beta}(v) \mathcal{B}_{\alpha}(u), \\
f(h) \mathcal{A}(u)= & \mathcal{A}(u) f(h), \quad f(h) \mathcal{B}_{i}(u)=\mathcal{B}_{i}(u) f(h+\hat{i}-\hat{1}), \\
f(h) \mathcal{D}_{i}^{j}(u)= & \mathcal{D}_{i}^{j}(u) f(h+\hat{i}-\hat{j})
\end{aligned}
$$

where the function $r, s$ are defined by

$$
r(u, \nu)=\frac{\sigma(u+w) \sigma(\nu w)}{\sigma(u) \sigma(\nu w+w)}, \quad s(u, \nu)=\frac{\sigma(w) \sigma(u+\nu w)}{\sigma(u) \sigma(\nu w-w)}, \quad \nu \in \mathbb{C} .
$$

Because of the simple poles in the functions $r, s$ at $u \in \mathbb{Z}+\tau \mathbb{Z}$, let us assume for convenience that all $\left\{v_{k}\right\}$ are distinct $\operatorname{modulo} \mathbb{Z}+\tau \mathbb{Z}$ and consider $u$ at a generic point. Take

$$
|\Omega\rangle=g(\lambda)|\Lambda\rangle
$$


as the so-called quasi-vacuum satisfying the following conditions

$$
\begin{aligned}
& \mathcal{A}(u)|\Omega\rangle=\frac{g(\lambda-\hat{1})}{g(\lambda)}|\Omega\rangle, \quad \mathcal{C}_{i}(u)|\Omega\rangle=0, \quad i=2, \cdots, n, \\
& \mathcal{D}_{i}^{j}(u)|\Omega\rangle=\delta_{j}^{i} D_{i}(u, \lambda) \frac{g(\lambda-\hat{i})}{g(\lambda)}|\Omega\rangle, \quad i, j=2, \cdots, n, \\
& f(h)|\Omega\rangle=f(N \hat{1})|\Omega\rangle, \quad \mathcal{B}_{i}(u)|\Omega\rangle \neq 0, \quad i=2, \cdots, n,
\end{aligned}
$$

where $D_{i}(u, \lambda), p_{k}$ and $q_{k}$ are given in (2.24) and (2.25). We will specify the function $g(\lambda)$ later (3.23)

Now, let us evaluate the action of $\mathcal{A}(u)$ on $\left|\lambda ;\left\{v_{k}\right\}\right\rangle$. Many terms will appear when we move $\mathcal{A}(u)$ from the left to the right of $\mathcal{B}_{i}\left(v_{k}\right)$ 's. They can be classified into two types: wanted and unwanted terms. The wanted terms in $\mathcal{A}(u)\left|\lambda ;\left\{v_{k}\right\}\right\rangle$ can be obtained by retaining the first term in the commutation relation (3.11). The unwanted terms arising from the second term of (3.11), have some $B\left(v_{k}\right)$ replaced by $B(u)$. One unwanted term where $B\left(v_{N 1}\right)$ is replaced by $B(u)$ can be obtained by using firstly the second terms of (3.11), then repeatedly using the first term of (3.11). Thanks to the commutation relation (3.13), one can easily obtain the other unwanted terms where $B\left(v_{k}\right)$ is replaced by $B(u)$. So, we can find the action of $A(u)$ on $\left|\lambda ;\left\{v_{k}\right\}\right\rangle$

$$
\begin{aligned}
& \mathcal{A}(u) F^{i_{1}, i_{2}, \cdots, i_{N_{1}}}\left(\lambda ;\left\{v_{k}\right\}\right) \mathcal{B}_{i_{N_{1}}}\left(v_{N_{1}}\right) \mathcal{B}_{i_{N_{1}-1}}\left(v_{N_{1}-1}\right) \cdots \mathcal{B}_{i_{1}}\left(v_{1}\right)|\Omega\rangle \\
& =F^{i_{1}, i_{2}, \cdots, i_{N_{1}}}\left(\lambda-\hat{1} ;\left\{v_{k}\right\}\right)\left\{\prod_{s=k+1}^{N_{1}} r\left(v_{k}-u,\left\langle\lambda-\hat{i}_{s}, \epsilon_{i_{k}}-\epsilon_{1}\right\rangle\right)\right\} \\
& \quad \times B_{i_{N_{1}}}\left(v_{N_{1}}\right) \cdots B_{i_{1}}\left(v_{1}\right) \frac{g(\lambda-\hat{1})}{g(\lambda)}|\Omega\rangle \\
& +F^{i_{1}, i_{2}, \cdots, i_{N_{1}}}\left(\lambda-\hat{1} ;\left\{v_{k}\right\}\right) s\left(u-v_{N_{1}}, \lambda_{1 i_{N_{1}}}\right)\left\{\prod_{k=1}^{N_{1}-1} r\left(v_{k}-v_{N_{1}},\left\langle\lambda-\sum_{s=k+1}^{N_{1}} \hat{i}_{s}, \epsilon_{i_{k}}-\epsilon_{1}\right\rangle\right)\right\} \\
& \quad \times B_{i_{N_{1}}}(u) B_{i_{N_{1}-1}}\left(v_{N_{1}-1}\right) \cdots B_{i_{1}}\left(v_{1}\right) \frac{g(\lambda-\hat{1})}{g(\lambda)}|\Omega\rangle \\
& \quad+\text { o.u.t. }
\end{aligned}
$$

where o.u.t. stands for the other unwanted terms. Due to the restriction condition (3.6) and $N_{1}=(n-1) \times l$, we have

$$
\left\{\prod_{k=1}^{N_{1}} r\left(v_{k}-u,\left\langle\lambda-\sum_{s=k+1}^{N_{1}} \hat{i}_{s}, \epsilon_{i_{k}}-\epsilon_{1}\right\rangle\right)\right\}=\left\{\prod_{k=1}^{N_{1}} \frac{\sigma\left(v_{k}-u+w\right)}{\sigma\left(v_{k}-u\right)}\right\}\left\{\prod_{j=2}^{n} \frac{\sigma\left(\lambda_{j 1} w-l w+w\right)}{\sigma\left(\lambda_{j 1} w+w\right)}\right\},
$$




$$
\sum_{k=1}^{N_{1}} \hat{i}_{k}=l \sum_{i=2}^{n} \hat{i}=-l \hat{1} .
$$

In order that the Bethe ansatz equation (3.46) and the eigenvalues (3.31) should be independent of $\lambda$, one needs to choose (cf. [10])

$$
g(\lambda)=e^{\left\langle\alpha^{(1)} \epsilon_{1}, \lambda w\right\rangle} \prod_{j=2}^{n}\left\{\prod_{k=1}^{l} \frac{\sigma\left(\lambda_{j 1} w+k w\right)}{\sigma(w)}\right\} .
$$

The action of $\mathcal{A}(u)$ on $\left|\lambda ;\left\{v_{k}\right\}\right\rangle$ becomes

$$
\begin{aligned}
& \mathcal{A}(u) F^{i_{1}, i_{2}, \cdots, i_{N_{1}}}\left(\lambda ;\left\{v_{k}\right\}\right) \mathcal{B}_{i_{N_{1}}}\left(v_{N_{1}}\right) \mathcal{B}_{i_{N_{1}-1}}\left(v_{N_{1}-1}\right) \cdots \mathcal{B}_{i_{1}}\left(v_{1}\right)|\Omega\rangle \\
& =e^{\frac{1-n}{n} \alpha^{(1)} w} \prod_{k=1}^{N_{1}} \frac{\sigma\left(v_{k}-u+w\right)}{\sigma\left(v_{k}-u\right)} F^{i_{1}, i_{2}, \cdots, i_{N_{1}}}\left(\lambda-\hat{1} ;\left\{v_{k}\right\}\right) B_{i_{N_{1}}}\left(v_{N_{1}}\right) \cdots B_{i_{1}}\left(v_{1}\right)|\Omega\rangle \\
& +s\left(u-v_{N_{1}}, \lambda_{1 i_{N_{1}}}\right) \frac{\sigma\left(\lambda_{i_{N_{1}}} w+w\right)}{\sigma\left(\lambda_{i_{N_{1}}} w\right)} e^{\frac{1-n}{n} \alpha^{(1)} w} \prod_{k=1}^{N_{1}-1} \frac{\sigma\left(v_{k}-v_{N_{1}}+w\right)}{\sigma\left(v_{k}-v_{N_{1}}\right)} \\
& \quad \times F^{i_{1}, i_{2}, \cdots, i_{N_{1}}}\left(\lambda-\hat{1} ;\left\{v_{k}\right\}\right) B_{i_{N_{1}}}(u) B_{i_{N_{1}-1}}\left(v_{N_{1}-1}\right) \cdots B_{i_{1}}\left(v_{1}\right)|\Omega\rangle \\
& + \text { o.u.t.. }
\end{aligned}
$$

Next, we evaluate the action of $\mathcal{D}_{i}^{i}(u)$ on $\left|\lambda ;\left\{v_{k}\right\}\right\rangle$ :

$$
\begin{aligned}
\mathcal{D}_{i}^{i}(u) & F^{i_{1}, i_{2}, \cdots, i_{N_{1}}}\left(\lambda ;\left\{v_{k}\right\}\right) \mathcal{B}_{i_{N_{1}}}\left(v_{N_{1}}\right) \mathcal{B}_{i_{N_{1}-1}}\left(v_{N_{1}-1}\right) \cdots \mathcal{B}_{i_{1}}\left(v_{1}\right)|\Omega\rangle \\
= & \left\{\prod_{k=1}^{N_{1}} r\left(u-v_{k},\left\langle\lambda-h-\left(N_{1}-k\right) \hat{1}, \epsilon_{i}-\epsilon_{1}\right\rangle\right)\right\} \\
& \times\left\{\prod_{k=1}^{m} \frac{\sigma\left(u-p_{k}\right)}{\sigma\left(u-q_{k}\right)}\right\} \frac{\sigma\left(\lambda_{i 1} w+(N-l) w\right)}{\sigma\left(\lambda_{i 1} w-l w\right)} \frac{g(\lambda-\hat{i}+l \hat{1})}{g(\lambda+l \hat{1})} \\
& \times\left\{L^{(1)}{ }_{i}^{i}(u, \lambda)\right\}_{i_{1}, i_{2}, \cdots, i_{N_{1}}}^{i_{1}^{\prime}, i_{2}^{\prime}, \cdots, i_{N_{1}}^{\prime}} F^{i_{1}, i_{2}, \cdots, i_{N_{1}}}\left(\lambda-\hat{i} ;\left\{v_{k}\right\}\right) \\
& \times \mathcal{B}_{i_{N_{1}}^{\prime}}\left(v_{N_{1}}\right) \mathcal{B}_{i_{N_{1}-1}^{\prime}}\left(v_{N_{1}-1}\right) \cdots \mathcal{B}_{i_{1}^{\prime}}\left(v_{1}\right)|\Omega\rangle \\
+ & \text { u.t., }
\end{aligned}
$$

where u.t. stands for the all unwanted terms. We have introduced the convenient notation

$$
\begin{aligned}
\left\{L_{i}^{(1)}(u, \lambda)\right\}_{i_{1}, i_{2}, \cdots, i_{N_{1}}}^{i_{1}^{\prime}, i_{2}^{\prime}, \cdots, i_{N_{1}}^{\prime}}= & R_{\gamma_{N_{1}-1}, i_{1}}^{j, i_{1}^{\prime}}\left(u-v_{1} ; \lambda-\sum_{k=2}^{N_{1}} \hat{i}_{k}^{\prime}\right) R_{\gamma_{N_{1}-2}, i_{2}}^{\gamma_{N_{1}-1}, i_{2}^{\prime}}\left(u-v_{2} ; \lambda-\sum_{k=3}^{N_{1}} \hat{i}_{k}^{\prime}\right) \\
& \times \cdots R_{i, i_{N_{1}}}^{\gamma_{1}, i_{N_{1}}^{\prime}}\left(u-v_{N_{1}} ; \lambda\right)
\end{aligned}
$$


and all the indices take the values $2, \cdots, n$. Noting the explicit form of the function $g(\lambda)$ given in (3.23) and the zero-weight of the state $\left|\lambda ;\left\{v_{k}\right\}\right\rangle$, the action of $\mathcal{D}_{i}^{i}(u)$ becomes

$$
\begin{aligned}
\mathcal{D}_{i}^{i}(u)\left|\lambda ;\left\{v_{k}\right\}\right\rangle & =e^{\frac{\alpha^{(1)}}{n}} w\left\{\prod_{k=1}^{N_{1}} \frac{\sigma\left(u-v_{k}+w\right)}{\sigma\left(u-v_{k}\right)}\right\}\left\{\prod_{k=1}^{m} \frac{\sigma\left(u-p_{k}\right)}{\sigma\left(u-q_{k}\right)}\right\} \\
& \times\left\{L^{(1)}{ }_{i}^{i}(u, \lambda)\right\}_{i_{1}, i_{2}, \cdots, i_{N_{1}}}^{i_{1}^{\prime}, i_{2}^{\prime}, \cdots, i_{N_{1}}^{\prime}} F^{i_{1}, i_{2}, \cdots, i_{N_{1}}}\left(\lambda-\hat{i} ;\left\{v_{k}\right\}\right) \\
& \times \mathcal{B}_{i_{N_{1}}^{\prime}}\left(v_{N_{1}}\right) \mathcal{B}_{i_{N_{1}-1}^{\prime}}\left(v_{N_{1}-1}\right) \cdots \mathcal{B}_{i_{1}^{\prime}}\left(v_{1}\right)|\Omega\rangle \\
- & e^{\frac{\alpha(1)}{n} w} s\left(u-v_{N_{1}}, \lambda_{1 i}\right) \frac{\sigma\left(\lambda_{i 1} w+w\right)}{\sigma\left(\lambda_{i 1} w\right)}\left\{\prod_{k=1}^{N_{1}-1} \frac{\sigma\left(v_{N_{1}}-v_{k}+w\right)}{\sigma\left(v_{N_{1}}-v_{k}\right)}\right\}\left\{\prod_{k=1}^{m} \frac{\sigma\left(v_{N_{1}}-p_{k}\right)}{\sigma\left(v_{N_{1}}-q_{k}\right)}\right\} \\
& \times\left\{L^{(1)}{ }_{i}^{i}\left(v_{N_{1}}, \lambda\right)\right\}_{i_{1}, i_{2}, \cdots, i_{N_{1}}}^{i_{1}^{\prime}, i_{2}^{\prime}, \cdots, i_{N_{1}}^{\prime}} F^{i_{1}, i_{2}, \cdots, i_{N_{1}}}\left(\lambda-\hat{i} ;\left\{v_{k}\right\}\right) \\
& \times \mathcal{B}_{i_{N_{1}}}(u) \mathcal{B}_{i_{N_{1}-1}^{\prime}}\left(v_{N_{1}-1}\right) \cdots \mathcal{B}_{i_{1}^{\prime}}\left(v_{1}\right)|\Omega\rangle \\
+ & \text { o.u.t.. }
\end{aligned}
$$

Keeping the initial condition of R-matrix (2.11) and the notation (3.26) in mind, we can find the action of the transfer matrices on the state $\left|\lambda ;\left\{v_{k}\right\}\right\rangle$ :

$$
\begin{aligned}
T(u) \mid & \left.\lambda ;\left\{v_{k}\right\}\right\rangle=\left(\mathcal{A}(u)+\sum_{i=2}^{n} \mathcal{D}_{i}^{i}(u)\right)\left|\lambda ;\left\{v_{k}\right\}\right\rangle \\
= & e^{\frac{1-n}{n} \alpha^{(1)} w} \prod_{k=1}^{N_{1}} \frac{\sigma\left(v_{k}-u+w\right)}{\sigma\left(v_{k}-u\right)} F^{i_{1}, i_{2}, \cdots, i_{N_{1}}}\left(\lambda-\hat{1} ;\left\{v_{k}\right\}\right) B_{i_{N_{1}}}\left(v_{N_{1}}\right) \cdots B_{i_{1}}\left(v_{1}\right)|\Omega\rangle \\
+ & e^{\frac{\alpha^{(1)}}{n} w}\left\{\prod_{k=1}^{N_{1}} \frac{\sigma\left(u-v_{k}+w\right)}{\sigma\left(u-v_{k}\right)}\right\}\left\{\prod_{k=1}^{m} \frac{\sigma\left(u-p_{k}\right)}{\sigma\left(u-q_{k}\right)}\right\} \\
& \times\left\{T^{(1)}(u)\right\}_{i_{1}, i_{2}, \cdots, i_{N_{1}}}^{i_{1}^{\prime}, F^{\prime}, \cdots, i^{\prime}}{ }^{i_{1}, i_{2}, \cdots, i_{N_{1}}}\left(\lambda ;\left\{v_{k}\right\}\right) \\
& \times \mathcal{B}_{i_{N_{1}}^{\prime}}\left(v_{N_{1}}\right) \mathcal{B}_{i_{N_{1}-1}^{\prime}}\left(v_{N_{1}-1}\right) \cdots \mathcal{B}_{i_{1}^{\prime}}\left(v_{1}\right)|\Omega\rangle \\
+ & s\left(u-v_{N_{1}}, \lambda_{1 i_{N_{1}}}\right) \frac{\sigma\left(\lambda_{i_{N_{1}}} w+w\right)}{\sigma\left(\lambda_{i_{N_{1}}} w\right)} e^{\frac{1-n}{n} \alpha^{(1)} w} \prod_{k=1}^{N_{1}-1} \frac{\sigma\left(v_{k}-v_{N_{1}}+w\right)}{\sigma\left(v_{k}-v_{N_{1}}\right)} \\
& \times F^{i_{1}, i_{2}, \cdots, i_{N_{1}}}\left(\lambda-\hat{1}_{;}\left\{v_{k}\right\}\right) B_{i_{N_{1}}}(u) B_{i_{N_{1}-1}}\left(v_{N_{1}-1}\right) \cdots B_{i_{1}}\left(v_{1}\right)|\Omega\rangle \\
- & \left(\sum_{i=2}^{n} e^{\frac{\alpha(1)}{n} w} s\left(u-v_{N_{1}}, \lambda_{1 i}\right) \frac{\sigma\left(\lambda_{i 1} w+w\right)}{\sigma\left(\lambda_{i 1} w\right)}\left\{\prod_{k=1}^{N_{1}-1} \frac{\sigma\left(v_{N_{1}}-v_{k}+w\right)}{\sigma\left(v_{N_{1}}-v_{k}\right)}\right\}\left\{\prod_{k=1}^{m} \frac{\sigma\left(v_{N_{1}}-p_{k}\right)}{\sigma\left(v_{N_{1}}-q_{k}\right)}\right\}\right. \\
& \times \delta_{i_{N_{1}}^{\prime}}^{i}\left\{T^{(1)}\left(v_{N_{1}}\right)\right\}_{i_{1}, i_{2}, \cdots, i_{N_{1}}}^{i_{1}^{\prime}, i_{2}^{\prime}, \cdots, i_{N_{1}}^{\prime}} F^{i_{1}, i_{2}, \cdots, i_{N_{1}}}\left(\lambda ;\left\{v_{k}\right\}\right) \\
& \left.\times \mathcal{B}_{i_{N_{1}}^{\prime}}(u) \mathcal{B}_{i_{N_{1}-1}^{\prime}}\left(v_{N_{1}-1}\right) \cdots \mathcal{B}_{i_{1}^{\prime}}\left(v_{1}\right)|\Omega\rangle\right)
\end{aligned}
$$




$$
+ \text { o.u.t. }
$$

where we have introduced the reduced transfer matrices $T^{(1)}(u)$

$$
T^{(1)}(u) F^{i^{\prime}, i_{2}^{\prime}, \cdots, i_{N_{1}}^{\prime}}\left(\lambda ;\left\{v_{k}\right\}\right)=\sum_{i=2}^{n} L_{i}^{(1)^{i}}(u, \lambda)_{i_{1}, i_{2}, \cdots, i_{N_{1}}}^{i_{1}^{\prime}, i_{2}^{\prime}, \cdots, i_{N_{1}}^{\prime}} F^{i_{1}, i_{2}, \cdots, i_{N_{1}}}\left(\lambda-\hat{i} ;\left\{v_{k}\right\}\right) .
$$

Thanks to the commutation relations (3.13), one can obtain the explicit expressions of all the o.u.t. The equation (3.28) tells that the state $\left|\lambda ;\left\{v_{k}\right\}\right\rangle$ is not an eigenvector of the transfer matrices $T(u)$ unless $F^{\prime} s$ are the eigenvectors of the reduced transfer matrices $T^{(1)}(u)$. The condition that the third and fourth terms in the above equation should cancel each other and also the all o.u.t. terms vanish, will give a restriction on the $N_{1}$ parameters $\left\{v_{k}\right\}$, the so-called Bethe ansatz equations. Hence we arrive at the final results:

$$
T(u)\left|\lambda ;\left\{v_{k}\right\}\right\rangle=t\left(u ;\left\{v_{k}\right\}\right)\left|\lambda ;\left\{v_{k}\right\}\right\rangle
$$

The eigenvalue reads

$$
\begin{aligned}
t\left(u ;\left\{v_{k}\right\}\right)=e^{(1-n) \bar{\alpha} w}\left\{\prod_{k=1}^{N_{1}} \frac{\sigma\left(v_{k}-u+w\right)}{\sigma\left(v_{k}-u\right)}\right\} \\
+e^{\bar{\alpha} w}\left\{\prod_{k=1}^{N_{1}} \frac{\sigma\left(u-v_{k}+w\right)}{\sigma\left(u-v_{k}\right)}\right\}\left\{\prod_{k=1}^{m} \frac{\sigma\left(u-p_{k}\right)}{\sigma\left(u-q_{k}\right)}\right\} t^{(1)}\left(u ;\left\{v_{k}^{(1)}\right\}\right),
\end{aligned}
$$

in which

$$
\begin{aligned}
& T^{(1)}(u) F^{i_{1}^{\prime}, i_{2}^{\prime}, \cdots, i_{N_{1}}^{\prime}}\left(\lambda ;\left\{v_{k}\right\}\right)=e^{-\frac{\sum_{i=2}^{n} \alpha^{(i)}}{n(n-1)} w} t^{(1)}\left(u ;\left\{v_{k}^{(1)}\right\}\right) F^{i_{1}^{\prime}, i_{2}^{\prime}, \cdots, i_{N_{1}}^{\prime}}\left(\lambda ;\left\{v_{k}\right\}\right), \\
& F^{i_{1}^{\prime}, i_{2}^{\prime}, \cdots, i_{N_{1}}^{\prime}}\left(\lambda-\hat{1} ;\left\{v_{k}\right\}\right)=e^{\frac{\sum_{i=2}^{n} \alpha^{(i)}}{n} w} F^{i_{1}^{\prime}, i_{2}^{\prime}, \cdots, i_{N_{1}}^{\prime}}\left(\lambda ;\left\{v_{k}\right\}\right),
\end{aligned}
$$

and $\left\{v_{k} \mid k=1,2, \cdots, N_{1}\right\}$ satisfy

$$
e^{-n \bar{\alpha} w}\left\{\prod_{k=1, k \neq s}^{N_{1}} \frac{\sigma\left(v_{k}-v_{s}+w\right)}{\sigma\left(v_{k}-v_{s}-w\right)}\right\}=\left\{\prod_{k=1}^{m} \frac{\sigma\left(v_{s}-p_{k}\right)}{\sigma\left(v_{s}-q_{k}\right)}\right\} t^{(1)}\left(v_{s} ;\left\{v_{k}^{(1)}\right\}\right) .
$$

The parameters $\left\{v_{k}^{(1)} \mid k=1,2, \cdots, N_{2}\right\}$ will be specified later by the Bethe ansatz equations (3.46).

The diagonalization of the transfer matrices $T(u)$ is now reduced to diagonalization of the reduced transfer matrices $T^{(1)}(u)$ in (3.32) . The explicit expression of $T^{(1)}(u)$ given in (3.29) 
implies that $T^{(1)}(u)$ can be considered as the transfer matrices of an $E_{\tau, \eta}\left(s l_{n-1}\right)$-module $W^{(1)}$ (or reduced space): $N_{1}$ tensor product of fundamental representations of $E_{\tau, \eta}\left(s l_{n-1}\right)$ with evaluation points $\left\{v_{k}\right\}$. We can use the same method to find the eigenvalue of $T^{(1)}(u)$ as we have done for the diagonalization of $T(u)$. Similarly to (3.1) - (3.2), we introduce

$$
\begin{aligned}
& \mathcal{A}^{(1)}(u)=\left\{\tilde{L}^{(1)}\right\}_{2}^{2}(u), \quad \mathcal{B}_{i}^{(1)}(u)=\left\{\tilde{L}^{(1)}\right\}_{i}^{2}(u), \quad i=3, \cdots, n, \\
& \mathcal{C}_{i}^{(1)}(u)=\left\{\tilde{L}^{(1)}\right\}_{2}^{i}(u), \quad \mathcal{D}_{i}^{(1)}{ }_{i}^{j}(u)=\left\{\tilde{L}^{(1)}\right\}_{i}^{j}(u), \quad i, j=3, \cdots, n .
\end{aligned}
$$

Then the reduced transfer matrices (3.29) can be rewritten

$$
T^{(1)}(u)=\mathcal{A}^{(1)}(u)+\sum_{i=3}^{n} \mathcal{D}^{(1)}{ }_{i}^{i}(u)
$$

We seek the common eigenvectors of the reduced transfer matrices $T^{(1)}(u)$ in an analogous form to (3.7):

$$
\left|\lambda ;\left\{v_{k}^{(1)}\right\}\right\rangle^{(1)}=\sum_{i_{1}, \cdots, i_{N_{2}}=3}^{n} F^{(1)^{i_{1}, i_{2}, \cdots, i_{N_{2}}}}\left(\lambda ;\left\{v_{k}^{(1)}\right\}\right) \mathcal{B}_{i_{N_{2}}}^{(1)}\left(v_{N_{2}}^{(1)}\right) \mathcal{B}_{i_{N_{2}-1}}^{(1)}\left(v_{N_{2}-1}^{(1)}\right) \cdots \mathcal{B}_{i_{1}}^{(1)}\left(v_{1}^{(1)}\right)\left|\Omega^{(1)}\right\rangle
$$

with the restriction of zero-weight conditions similar to (3.6) . The quasi-vacuum $\left|\Omega^{(1)}\right\rangle$ is the corresponding highest weight vector in the reduced space $W^{(1)}$. We can also derive the relevant commutation relations among $\mathcal{A}^{(1)}(u), \mathcal{D}^{(1)}{ }_{i}^{j}$ and $B_{i}^{(1)}(u)(i, j=3,4, \cdots, n)$ similar to (3.11) - (3.15). Using the same method as we have done for the diagonalization of $T(u)$, we obtain the eigenvalue of the reduced transfer matrices $T^{(1)}(u)$ :

$$
T^{(1)}(u)\left|\lambda ;\left\{v_{k}^{(1)}\right\}\right\rangle^{(1)}=t^{(1)}\left(u ;\left\{v_{k}^{(1)}\right\}\right)\left|\lambda ;\left\{v_{k}^{(1)}\right\}\right\rangle^{(1)} .
$$

Though very complicated, the coefficients $F^{i_{1}, i_{2}, \cdots, i_{N_{1}}}\left(\lambda ;\left\{v_{k}\right\}\right)$ could be extracted from the eigenvectors $\left|\lambda ;\left\{v_{k}^{(1)}\right\}\right\rangle^{(1)}$, in principle. The eigenvalue $t^{(1)}\left(u ;\left\{v_{k}^{(1)}\right\}\right)$ is given by

$$
\begin{aligned}
t^{(1)}\left(u ;\left\{v_{k}^{(1)}\right\}\right)=e^{(2-n) \bar{\alpha}^{(1)} w} & \left\{\prod_{k=1}^{N_{2}} \frac{\sigma\left(v_{k}^{(1)}-u+w\right)}{\sigma\left(v_{k}^{(1)}-u\right)}\right\} \\
+e^{\bar{\alpha}^{(1)}} w & \left\{\prod_{k=1}^{N_{2}} \frac{\sigma\left(u-v_{k}^{(1)}+w\right)}{\sigma\left(u-v_{k}^{(1)}\right)}\right\}\left\{\prod_{k=1}^{N_{1}} \frac{\sigma\left(u-v_{k}\right)}{\sigma\left(u-v_{k}+w\right)}\right\} t^{(2)}\left(u ;\left\{v_{k}^{(2)}\right\}\right) .
\end{aligned}
$$


The parameters $\left\{v_{k}^{(2)} \mid k=1,2, \cdots, N_{3}\right\}$ will be specified later by the Bethe ansatz equations (3.46) . The function $t^{(2)}\left(u ;\left\{v_{k}^{(2)}\right\}\right)$ is the eigenvalue of the second reduced transfer matrices $T^{(2)}(u)$

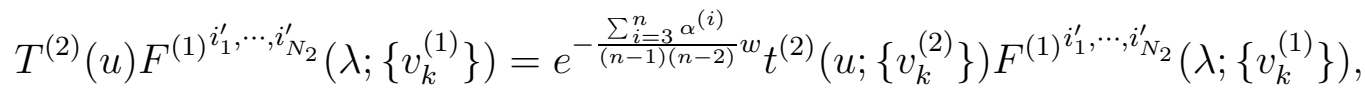

and $T^{(2)}(u)$ is given by (3.26) and (3.29) with all indices taking values over $3, \cdots, n$ and depending on $\left\{v_{k}^{(1)}\right\}$ instead of $\left\{v_{k}\right\}$. Hence, the diagonalization of $T(u)$ is further reduced to the diagonalization of $T^{(2)}(u)$ in (3.41). This is so-called nested Bethe ansatz. Repeating the above procedure further $n-2$ times, one can reduce to the last reduced transfer matrices $T^{(n-1)}(u)$ which is trivial to get the eigenvalues. At the same time we need to introduce the $\frac{n(n-1)}{2} l$ parameters $\left\{\left\{v_{k}^{(i)} \mid k=1,2, \cdots, N_{i+1}\right\}, i=0,1, \cdots, n-2\right\}$ to specify the eigenvectors of the corresponding reduced transfer matrices $T^{(i)}(u)$ (including the original one $T(u)=$ $T^{(0)}(u)$ ), and $n$ arbitrary complex numbers $\left\{\alpha^{(i)} \mid i=1 \cdots, n\right\}$ to specify the quasi-vacuum $\left|\Omega^{(i)}\right\rangle$ of each step as in (3.16) and (3.23). Finally, we obtain all the eigenvalues of the reduced transfer matrices $T^{(i)}(u)$ with the eigenvalue $t^{(i)}\left(u ;\left\{v_{k}^{(i)}\right\}\right)$ in a recurrence form

$$
\begin{aligned}
& t^{(i)}\left(u ;\left\{v_{k}^{(i)}\right\}\right)=e^{(i+1-n) \bar{\alpha}^{(i)} w}\left\{\prod_{k=1}^{N_{i+1}} \frac{\sigma\left(v_{k}^{(i)}-u+w\right)}{\sigma\left(v_{k}^{(i)}-u\right)}\right\} \\
&+e^{\bar{\alpha}^{(i)} w}\left\{\prod_{k=1}^{N_{i+1}} \frac{\sigma\left(u-v_{k}^{(i)}+w\right)}{\sigma\left(u-v_{k}^{(i)}\right)}\right\}\left\{\prod_{k=1}^{N_{i}} \frac{\sigma\left(u-p_{k}^{(i)}\right)}{\sigma\left(u-q_{k}^{(i)}\right)}\right\} t^{(i+1)}\left(u ;\left\{v_{k}^{(i+1)}\right\}\right), \\
& i=0,1, \cdots, n-2, \\
& t^{(0)}\left(u ;\left\{v_{k}^{(0)}\right\}\right)=t\left(u ;\left\{v_{k}\right\}\right), t^{(n-1)}(u)=1,
\end{aligned}
$$

where $\bar{\alpha}^{(i)}, i=0,1, \cdots, n-2$ are given by (3.10) $, \bar{\alpha}=\alpha^{\overline{(0)}}, N_{0}=m$ and

$$
\begin{aligned}
& p_{k}^{(0)}=p_{k}=z_{k}, \quad q_{k}^{(0)}=q_{k}=z_{k}-l_{k} w, \quad k=1,2, \cdots, m, \\
& p_{k}^{(i)}=v_{k}^{(i-1)}, \quad q_{k}^{(i)}=v_{k}^{(i-1)}-w, \quad i=1,2, \cdots, n-2, \quad k=1,2, \cdots, N_{i} .
\end{aligned}
$$

The $\left\{\left\{v_{k}^{(i)}\right\}\right\}$ satisfy the following Bethe ansatz equations

$$
e^{(i-n) \bar{\alpha}^{(i)} w}\left\{\prod_{k=1, k \neq s}^{N_{i+1}} \frac{\sigma\left(v_{k}^{(i)}-v_{s}^{(i)}+w\right)}{\sigma\left(v_{k}^{(i)}-v_{s}^{(i)}-w\right)}\right\}=\left\{\prod_{k=1}^{N_{i}} \frac{\sigma\left(v_{s}^{(i)}-p_{k}^{(i)}\right)}{\sigma\left(v_{s}^{(i)}-q_{k}^{(i)}\right)}\right\} t^{(i+1)}\left(v_{s}^{(i)} ;\left\{v_{k}^{(i+1)}\right\}\right) .
$$

We conclude this section with some remarks on analytic properties of the functions $t^{(i)}\left(u ;\left\{v_{k}^{(i)}\right\}\right)$. By construction, the eigenvalue functions $t^{(i)}\left(u ;\left\{v_{k}^{(i)}\right\}\right)$ of the transfer matrices should not be singular at $u=v_{k}^{(i)}(\operatorname{modulo} \mathbb{Z}+\tau \mathbb{Z})$ with $0 \leq i \leq n-2,1 \leq k \leq N_{i+1}$. 
On the other hand, the Bethe ansatz equations (3.34) and (3.46) are derived from the requirement that the unwanted terms should vanish. It is interesting to note that these constraints could be understood from a different point of view: from equation (3.31), we know that $u=v_{k}^{(0)}=v_{k}(\operatorname{modulo} \mathbb{Z}+\tau \mathbb{Z})$ is a possible simple pole position of $t\left(u ;\left\{v_{k}\right\}\right)$. However, the constraints on $\left\{v_{k}\right\}$, the Bethe ansatz equations (3.34) simply tell that the residue of $t\left(u ;\left\{v_{k}\right\}\right)$ at $u=v_{k}(\operatorname{modulo} \mathbb{Z}+\tau \mathbb{Z})$ is vanishing. Hence, $t\left(u ;\left\{v_{k}\right\}\right)$ is analytic at $u=v_{k}$ $(\operatorname{modulo} \mathbb{Z}+\tau \mathbb{Z})$. Similarly, the Bethe ansatz equations (3.46) ensure the analyticity of $t^{(i)}\left(u ;\left\{v_{k}^{(i)}\right\}\right)$ at all $u=v_{k}^{(i)}(\operatorname{modulo} \mathbb{Z}+\tau \mathbb{Z})$. Therefore, the eigenvalues of transfer matrices $T(u)$ are analytic functions of $u$.

\section{The $\mathbb{Z}_{n}$ Belavin model}

We shall show in this section how to obtain the eigenvectors and the corresponding eigenvalues of the transfer matrices for $\mathbb{Z}_{n}$ Belavin model with periodic boundary condition from our results in section 3 .

The $\mathbb{Z}_{n}$ Belavin model [17] is based on the following R-matrix

$$
R_{B}(u)=\sum_{i, j, k, l} R_{i j}^{k l}(u) E_{i k} \otimes E_{l j}
$$

The coefficient functions are 18

$$
R_{i j}^{k l}(u)= \begin{cases}\frac{h(u) \sigma(w) \theta^{(i-j)}(u+w)}{\sigma(u+w) \theta^{(i-k)}(w) \theta^{(k-j)}(u)} & \text { if } i+j=k+l \bmod n \\ 0 & \text { otherwise }\end{cases}
$$

where

$$
h(u)=\frac{\prod_{j=0}^{n-1} \theta^{(j)}(u)}{\prod_{j=1}^{n-1} \theta^{(j)}(0)} .
$$

The R-matrix satisfies the quantum Yang-Baxter equation

$$
R_{12}\left(u_{1}-u_{2}\right) R_{13}\left(u_{1}-u_{3}\right) R_{23}\left(u_{2}-u_{3}\right)=R_{23}\left(u_{2}-u_{3}\right) R_{13}\left(u_{1}-u_{3}\right) R_{12}\left(u_{1}-u_{2}\right),
$$

and the initial condition: $R_{i j}^{k l}(0)=\delta_{i}^{l} \delta_{j}^{k}$. For generic evaluation points $z_{1}, \cdots, z_{m}$, one then defines the commuting transfer matrices

$$
\begin{aligned}
& T_{B}(u)=\operatorname{tr}_{0} L_{B}(u), \\
& L_{B}(u)=\left\{R_{B}\right\}_{01}\left(u-z_{1}\right) \cdots\left\{R_{B}\right\}_{0 m}\left(u-z_{m}\right),
\end{aligned}
$$


where the L-operator $L_{B}(u)$ acts on $\mathbb{C}^{n} \otimes\left(\mathbb{C}^{n}\right)^{\otimes m}$ and the transfer matrices $T_{B}(u)$ act on $\left(\mathbb{C}^{n}\right)^{\otimes m}$.

Using the intertwiner introduced by Jimbo et al [18, we can define the matrix $S(z, \lambda)$ with the elements $S_{j}^{i}(z, \lambda)=\frac{\theta^{(i)}\left(z+n w \lambda_{j}\right)}{\prod_{k \neq j} \sigma\left(\lambda_{k j} w\right)}$. When $\lambda \in \mathbb{P}_{\xi}$, the inverse matrix of $S(z, \lambda)$ exists. Then, one finds the following twisting relation holds:

$$
\begin{aligned}
& \left\{R_{B}\right\}_{12}\left(u_{1}-u_{2}\right) S\left(u_{1}, \lambda\right)^{(1)} S\left(u_{2}, \lambda-h^{(1)}\right)^{(2)} \\
& =S\left(u_{2}, \lambda\right)^{(2)} S\left(u_{1}, \lambda-h^{(2)}\right)^{(1)} R_{12}\left(u_{1}-u_{2}, \lambda\right)
\end{aligned}
$$

in which $R(u, \lambda)$ is defined by (2.7), (2.8) and (2.9). The modified quantum Yang-Baxter equation (2.10) and the quantum Yang-Baxter equation (4.4) are equivalent to each other due to the above twisting relation (4.7). Let us introduce

$$
S_{m}\left(z_{1}, \cdots, z_{m} ; \lambda\right)=S\left(z_{m}, \lambda\right)^{(m)} S\left(z_{m-1}, \lambda-h^{(m)}\right)^{(m-1)} \cdots S\left(z_{1}, \lambda-\sum_{k=2}^{m} h^{(k)}\right)^{(1)} .
$$

For the special case of $l_{1}=l_{2}=\cdots=l_{m}=1$, we find that the L-operator $L(u, \lambda)$ corresponding to the $E_{\tau, \eta}\left(s l_{n}\right)$-module $W$ is equivalent to the L-operator $L_{B}(u)$ of $\mathbb{Z}_{n}$ Belavin model (4.6) by the twisting relation

$$
\begin{aligned}
& S_{m}\left(z_{1}, \cdots, z_{m} ; \lambda\right) S(u, \lambda-h)^{(0)} L(u, \lambda) \\
& \quad=L_{B}(u) S(u, \lambda)^{(0)} S_{m}\left(z_{1}, \cdots, z_{m} ; \lambda-h^{(0)}\right) .
\end{aligned}
$$

In this formula $S_{m}\left(z_{1}, \cdots, z_{m} ; \lambda\right)$ acts on the factors from 1 to $m$, and $h=h^{(1)}+\cdots+h^{(m)}$. Acting the both sides of (4.9) on a state $e_{i} \otimes v$ with $v \in W[0]$, replacing $\lambda$ by $\lambda+\hat{i}$, and noting (2.14), we find

$$
\begin{gathered}
\sum_{j=1}^{n}\left(S(u, \lambda+\hat{j}) e_{j}\right) \otimes\left(S_{m}\left(z_{1}, \cdots, z_{m} ; \lambda+\hat{i}\right) L_{i}^{j}(u, \lambda+\hat{i}) v\right) \\
=L_{B}(u)\left\{\left(S(u, \lambda+\hat{i}) e_{i}\right) \otimes\left(S_{m}\left(z_{1}, \cdots, z_{m} ; \lambda\right) v\right)\right\}
\end{gathered}
$$

Therefore, one can derive

$$
\left.T_{B}(u) S_{m}\left(z_{1}, \cdots, z_{m} ; \lambda\right)\right|_{H}=\left.\sum_{i=1}^{n} S_{m}\left(z_{1}, \cdots, z_{m} ; \lambda+\hat{i}\right) L_{i}^{i}(u, \lambda+\hat{i})\right|_{H},
$$

where $H=F u n(W)[0]$ is preserved by the transfer matrices. 
Let $f \longmapsto \int f(\lambda)$ be a linear functional on the space $\mathcal{F}$ of functions of $\lambda \in \mathbb{C}^{n}$, such that $\int f(\lambda+\hat{i})=\int f(\lambda), i=1, \cdots, n$, for all $f \in \mathcal{F}$. Extend $\int$ to vector-valued functional by acting component-wise. Then for each eigenfunction $\Psi(\lambda)$ of $T(u)$ with eigenvalue $t(u)$, the transfer matrices of $\mathbb{Z}_{n}$ Belavin model $T_{B}(u)$ act on the vector $\int S_{m}\left(z_{1}, \cdots, z_{m} ; \lambda\right) \Psi(\lambda)$

$$
\begin{aligned}
T_{B}(u) & \int S_{m}\left(z_{1}, \cdots, z_{m} ; \lambda\right) \Psi(\lambda)=\sum_{i=1}^{n} \int S_{m}\left(z_{1}, \cdots, z_{m} ; \lambda+\hat{i}\right) L_{i}^{i}(u, \lambda+\hat{i}) \Psi(\lambda) \\
= & \sum_{i=1}^{n} \int S_{m}\left(z_{1}, \cdots, z_{m} ; \lambda\right) L_{i}^{i}(u, \lambda) \Psi(\lambda-\hat{i}) \\
= & \int S_{m}\left(z_{1}, \cdots, z_{m} ; \lambda\right) T(u) \Psi(\lambda)=t(u) \int S_{m}\left(z_{1}, \cdots, z_{m} ; \lambda\right) \Psi(\lambda) .
\end{aligned}
$$

Namely, the vector $\int S_{m}\left(z_{1}, \cdots, z_{m} ; \lambda\right) \Psi(\lambda)$ is an eigenvector of $T_{B}(u)$ with the same eigenvalue. Fortunately, such a linear functional on $\mathcal{F}$ has been given in [14, 10] for XYZ spin chain $\left(\mathbb{Z}_{2}\right.$-Belavin model) and its higher spin generalizations [16], for the generic $\mathbb{Z}_{n}$ Belavin model [15]. And our results recover those of [10] for $n=2$ case and coincide with those of [15] for $\mathbb{Z}_{n}$ Belavin model.

As in [16], one can construct the higher spin generalization of $\mathbb{Z}_{n}$ Belavin model by putting at each site a local $L$-operator

$$
L_{k}\left(u ; l_{k}\right)=\sum_{\alpha \in Z_{n}^{2}} \frac{\sigma_{\alpha}\left(u-z_{k}+\frac{w}{n}\right)}{\sigma_{\alpha}\left(\frac{w}{n}\right)} I_{\alpha} \otimes \rho_{l_{k}}\left\{\mathcal{S}_{\alpha}\right\}, \quad l_{k} \in \mathbb{Z}^{+}, \quad \mathcal{S}_{\alpha} \in S K,
$$

where $\rho_{l_{k}}$ is the spin- $l_{k}$ representation of $\mathbb{Z}_{n}$ Sklyanin algebra $S K$ with dimension $\frac{\left(n+l_{k}-1\right) !}{(n-1) ! l_{k} !}$ 12. The corresponding transfer matrices of the model are given by

$$
T_{B}\left(u ;\left\{l_{k}\right\}\right)=\operatorname{tr}_{0}\left\{L_{1}\left(u ; l_{1}\right) L_{2}\left(u ; l_{2}\right) \cdots L_{m}\left(u ; l_{m}\right)\right\} .
$$

Then, our construction in Sect.3 actually results in the eigenvalues formulation (3.31) of the transfer matrices (4.14), with the Bethe ansatz equations (3.46). In particular, our result recovers that of [16 when $n=2$.

\section{The elliptic $A_{n-1}$ Ruijsenaars-Schneider model}

If we take $E_{\tau, \eta}\left(s l_{n}\right)$-module $W$ for the special case ${ }^{1}$

$$
W=V_{\Lambda^{\left(l_{1}\right)}}\left(z_{1}\right), \quad \Lambda^{\left(l_{1}\right)}=l_{1} \Lambda_{1}=N \Lambda_{1}=n \times l \Lambda_{1},
$$

\footnotetext{
${ }^{1}$ Billey used algebraic Bethe ansatz method over the $E_{\tau, \eta}\left(s l_{n}\right)$-module $W=V_{\Lambda^{(1)}}\left(z_{1}\right) \otimes \cdots V_{\Lambda^{(1)}}\left(z_{1}-\right.$ $(n l-1) w)$ to obtain eigenvalues of elliptic N-body Ruijsenaars operator [1]
} 
the zero-weight space of this module is one-dimensional and the associated transfer matrices can be given by 9

$$
T(u)=\frac{\sigma\left(u-z_{1}+l w\right)}{\sigma\left(u-z_{1}+n l w\right)} M
$$

The operator $M$ is independent of $u$ and is given by

$$
M=\sum_{i=1}^{n}\left\{\prod_{j \neq i} \frac{\sigma\left(\lambda_{i j} w+l w\right)}{\sigma\left(\lambda_{i j} w\right)} \Gamma_{i}\right\} .
$$

Here $\left\{\Gamma_{i}\right\}$ are elementary difference operators: $\Gamma_{i} f(\lambda)=f(\lambda-\hat{i})$. In fact, for a special choice of the parameters [19], this difference operator $M$ is the Hamiltonian of elliptic $A_{n-1}$ type Ruijsenaars-Schneider model [20] with the special coupling constant $\gamma=l w$, up to conjugation by a function [13, 21]. Now, we consider the spectrum of $M$. The results of Sect. 3, enable us to obtain the spectrum of the Hamiltonian of the elliptic $A_{n-1}$ RuijsenaarsSchneider model as well as the eigenfunctions, in terms of the associated transfer matrices (5.2). Since $M$ is independent of $u$, we can evaluate the eigenvalue of $T(u)$ at a special value of $u, u=z_{1}$. Then the expression of the eigenvalue $t\left(u ;\left\{v_{k}\right\}\right)$ simplifies drastically, for the second term in the right hand side of (3.31) (the one depending on the eigenvalue of the reduced transfer matrices $\left.t^{(1)}\left(u ;\left\{v_{k}^{(1)}\right\}\right)\right)$ vanishes because $u-p_{1}^{(0)}=0$ in the case (5.8). Finally, we obtain the eigenvalues of $M$ :

$$
e^{(1-n) \bar{\alpha} w} \frac{\sigma(n l w)}{\sigma(l w)}\left\{\prod_{k=1}^{(n-1) \times l} \frac{\sigma\left(v_{k}-z_{1}+w\right)}{\sigma\left(v_{k}-z_{1}\right)}\right\}
$$

where $\left\{v_{k}\right\}$ and $\left\{\left\{v_{k}^{(i)}\right\}\right\}$ are determined by the nested Bethe ansatz equations

$$
\begin{aligned}
& e^{(i-n) \bar{\alpha}^{(i)} w}\left\{\prod_{k=1, k \neq s}^{N_{i+1}} \frac{\sigma\left(v_{k}^{(i)}-v_{s}^{(i)}+w\right)}{\sigma\left(v_{k}^{(i)}-v_{s}^{(i)}-w\right)}\right\}=\left\{\prod_{k=1}^{N_{i}} \frac{\sigma\left(v_{s}^{(i)}-p_{k}^{(i)}\right)}{\sigma\left(v_{s}^{(i)}-q_{k}^{(i)}\right)}\right\} t^{(i+1)}\left(v_{s}^{(i)} ;\left\{v_{k}^{(i+1)}\right\}\right), \\
& i=0,1, \cdots, n-2 .
\end{aligned}
$$

The functions $t^{(i)}\left(u ;\left\{v_{k}^{(i)}\right\}\right)$ appearing in (15.5) are defined by the following recurrence relations

$$
\begin{aligned}
& t^{(i)}\left(u ;\left\{v_{k}^{(i)}\right\}\right)=e^{(i+1-n) \bar{\alpha}^{(i)} w}\left\{\prod_{k=1}^{N_{i+1}} \frac{\sigma\left(v_{k}^{(i)}-u+w\right)}{\sigma\left(v_{k}^{(i)}-u\right)}\right\} \\
& +e^{\bar{\alpha}^{(i)} w}\left\{\prod_{k=1}^{N_{i+1}} \frac{\sigma\left(u-v_{k}^{(i)}+w\right)}{\sigma\left(u-v_{k}^{(i)}\right)}\right\}\left\{\prod_{k=1}^{N_{i}} \frac{\sigma\left(u-p_{k}^{(i)}\right)}{\sigma\left(u-q_{k}^{(i)}\right)}\right\} t^{(i+1)}\left(u ;\left\{v_{k}^{(i+1)}\right\}\right), \\
& i=1, \cdots, n-2, \\
& t^{(n-1)}(u)=1
\end{aligned}
$$


where $\bar{\alpha}^{(i)}, i=0,1, \cdots, n-2$ are given by (3.10), $\bar{\alpha}=\bar{\alpha}^{(0)}, N_{0}=1$ and

$$
\begin{aligned}
& p_{1}^{(0)}=z_{1}, \quad q_{1}^{(0)}=z_{1}-n l w, \\
& p_{k}^{(i)}=v_{k}^{(i-1)}, \quad q_{k}^{(i)}=v_{k}^{(i-1)}-w, \quad i=1,2, \cdots, n-2, \quad k=1,2, \cdots, N_{i} .
\end{aligned}
$$

\section{Conclusions}

We have studied some modules over the elliptic quantum group associated with $A_{n-1}$ type root systems. There are elliptic deformation of symmetric tensor product of the fundamental vector representation of $A_{n-1}$ (we have called it higher spin representation). Using the nested Bethe ansatz method, we exactly diagonalize the commuting transfer matrices associated with the tensor product of the $E_{\tau, \eta}\left(s l_{n}\right)$-modules with generic evaluation points. The eigenvalues of the transfer matrices and associated Bethe ansatz equation are given by (3.31) and (3.46) . For the special case of $n=2$, our result recovers that of Felder et al [10].

We also take the applications of our construction to some integrable models. For $l_{1}=$ $l_{2}=\cdots=1$ case, our result gives the spectrum problem of $\mathbb{Z}_{n}$ Belavin model with periodic boundary condition, which coincides with the result [15]. For the special case $W=V_{\Lambda^{(n l)}}\left(z_{1}\right)$, or equivalently $l_{1}=\cdots=l_{n l}=1$ and $z_{i}=z_{1}-(i-1) w$, our result gives the spectrum problem of the elliptic $A_{n-1}$ type Ruijsenaars operators, which is "relativistic" generalization [22] of the Calogero-Moser type integrable differential operators, with the special coupling constant $\gamma=l w$, which coincides with the result [11]. Moreover, for the generic case, our result gives eigenvalues of the transfer matrices given by (4.14) associated with higher spin generalization of $\mathbb{Z}_{n}$ Belavin model with periodic boundary condition, and it further enables us to construct the eigenvalues of all types of Ruijsenaars operators associated with the $A_{n-1}$ root system in the Bethe ansatz form 19.

\section{Acknowledgements}

We thank professors A. Belavin, S. Odake and K.J. Shi for their useful discussion. This work is supported in part by Grant-in-Aid for Scientific Research from the Ministry of Education, Culture, Sports, Science and Technology, No.12640261. W.-L. Yang is supported by the Japan Society for the Promotion of Science. 


\section{Appendix A: The commutation relations}

The starting point for deriving the commutation relations among $\mathcal{A}(u), \mathcal{D}_{i}^{j}(u)$ and $\mathcal{B}_{i}(u)$ $(i, j=2, \cdots, n)$ is the exchange relations (2.20) and (2.21). We rewrite (2.20) in the component form

$$
\sum_{i^{\prime}, j^{\prime}=1}^{n} R_{i^{\prime} j^{\prime}}^{a b}(u-v, \lambda-h) \tilde{L}_{c}^{i^{\prime}}(u) \tilde{L}_{d}^{j^{\prime}}(v)=\sum_{i^{\prime}, j^{\prime}=1}^{n} \tilde{L}_{j^{\prime}}^{b}(v) \tilde{L}_{i^{\prime}}^{a}(u) R_{c d}^{i^{\prime} j^{\prime}}(u-v, \lambda) .
$$

For $a=b=d=1, c=i \neq 1$, we obtain

$$
\begin{aligned}
\mathcal{A}(v) \mathcal{B}_{i}(u) & =\frac{1}{R_{i 1}^{i 1}(u-v, \lambda-\hat{1}-\hat{i})} \mathcal{B}_{i}(u) \mathcal{A}(v)-\frac{R_{i 1}^{1 i}(u-v, \lambda-\hat{1}-\hat{i})}{R_{i 1}^{i 1}(u-v, \lambda-\hat{1}-\hat{i})} \mathcal{B}_{i}(v) \mathcal{A}(u) \\
& =\frac{1}{R_{i 1}^{i 1}(u-v, \lambda)} \mathcal{B}_{i}(u) \mathcal{A}(v)-\frac{R_{i 1}^{1 i}(u-v, \lambda)}{R_{i 1}^{i 1}(u-v, \lambda)} \mathcal{B}_{i}(v) \mathcal{A}(u)
\end{aligned}
$$

by noting the definitions (2.8) and (2.9). The commutation relation (3.11) is a simple consequence of the above equation.

Similarly, for $a=j \neq 1, b=1, c=i \neq 1, d=l \neq 1$, we obtain

$$
\mathcal{D}_{i}^{j}(u) \mathcal{B}_{l}(v)=\sum_{\alpha, \beta=2}^{n}\left\{\frac{R_{i l}^{\alpha \beta}(u-v, \lambda)}{R_{j 1}^{j 1}(u-v, \lambda-h)} \mathcal{B}_{\beta}(v) \mathcal{D}_{\alpha}^{j}(v)\right\}-\frac{R_{1 j}^{j 1}(u-v, \lambda-h)}{R_{j 1}^{j 1}(u-v, \lambda-h)} \mathcal{B}_{i}(u) \mathcal{D}_{l}^{j}(v),
$$

from which follows the commutation relation (3.12). For $a=b=1, c=i \neq 1, d=j \neq 1$, we obtain

$$
\begin{aligned}
\mathcal{B}_{i}(u) \mathcal{B}_{j}(v) & =\sum_{\alpha, \beta=2}^{n} \mathcal{B}_{\beta}(v) \mathcal{B}_{\alpha}(u) R_{i j}^{\alpha \beta}(u-v, \lambda)=\sum_{\alpha, \beta=2}^{n} R_{i j}^{\alpha \beta}(u-v, \lambda-\hat{\alpha}-\hat{\beta}) \mathcal{B}_{\beta}(v) \mathcal{B}_{\alpha}(u) \\
& =\sum_{\alpha, \beta=2}^{n} R_{i j}^{\alpha \beta}(u-v, \lambda) \mathcal{B}_{\beta}(v) \mathcal{B}_{\alpha}(u)
\end{aligned}
$$

which leads to the commutation relation (3.13). The other commutation relations (3.14)(3.15) are derived from (2.21).

\section{References}

[1] L. A. Takhtajan and L. D. Faddeev, Russ. Math. Surv. 34 (1979), 11.

[2] H. B. Thacker, Rev. Mod. Phys. 53 (1982), 253. 
[3] V. E. Korepin, N. M. Bogoliubov and A. G. Izergin, Quantum Inverse Scattering Method and correlation Function, Cambridge Univ. Press, Cambridge, 1993.

[4] O. Babelon, H. J. de Vega and C. M. Viallet, Nucl. Phys. B200 (1982), 266.

[5] C. L. Schultz, Physica A122 (1983), 71.

[6] E. K. Sklyanin, Func. Anal. Appl. 17 (1983), 273.

[7] B. Y. Hou and H. Wei, J. Math. Phys. 30 (1989), 2750.

[8] G. Felder, Elliptic quantum groups, Proceedings of the International Congress of Mathematical Physics, Paris 1994, 211, International Press 1995.

[9] G. Felder and A. Varchenko, Commun. Math. Phys. 181 (1996), 741; J. Stat. Phys. 89 (1997), 963.

[10] G. Felder and A. Varchenko, Nucl. Phys. B480 (1996), 485.

[11] E. Billey, Algebraic nested Bethe ansatz for the elliptic Ruijsenaars model, math.QA/9806068.

[12] B. Y. Hou, K. J. Shi, W.-L. Yang, Z. X. Yang and S. Y. Zhou, Inter. J. Mod. Phys. A12 (1997), 2927.

[13] K. Hasegawa, Commun. Math. Phys. 187 (1997), 289.

[14] R. J. Baxter, Ann. Phys. (N.Y.) 76 (1973), 1, 25, 48.

[15] B. Y. Hou, M. L. Yan and Y. K. Zhou, Nucl. Phys. B324 (1989), 715.

[16] T. Takebe, J. Phys. A25 (1992), 1071.

[17] A. A. Belavin, Nucl. Phys. B180 (1981), 189.

[18] M. Jimbo, T. Miwa and M. Okado, Lett. Math. Phys. 14 (1987), 123; Nucl. Phys. B300 (1988), 74 .

[19] B. Y. Hou, R. Sasaki and W.-L. Yang, Eigenvalues of Ruijsenaars-Schneider model associated with $A_{n-1}$ root system in the Bethe ansatz formalism, YITP-03-13. 
[20] S. N. M. Ruijsenaars and H. Schneider, Ann. Phys. (N.Y.) 170 (1986), 370; Commun. Math. Phys. 110 (1987), 191.

[21] B. Y. Hou and W.-L. Yang, Phys. Lett. 261 (1999), 259; J. Math. Phys. 41 (2000), 357.

[22] H. Braden and R. Sasaki, Prog. Theor. Phys. 97 (1997), 1003. 